\title{
Traumatic Thoracic Aortic Dissection with Oral Administration of Antiplatelet Drugs in Multiple Trauma :
}

A Case Report

\author{
Takashi Ogino, ${ }^{1}$ Tetsushi Ogawa, ${ }^{2}$ Tetsuya Ishikawa, ${ }^{1}$ \\ Toshiyuki Tanaka, ${ }^{2}$ Masaaki Aiba, ${ }^{2}$ Ichiro Sakamoto, ${ }^{2}$ \\ Hisashi Shimizu, ${ }^{2}$ Hiroyuki Toya, ${ }^{2}$ Hotaka Yamazaki, ${ }^{2}$ \\ Yoko Motegi, ${ }^{2}$ Kazuhiro Sakata, ${ }^{3}$ Toshiharu Yamagishi, ${ }^{3}$ \\ Masayuki Sugano, ${ }^{4}$ Jun Atsumi, ${ }^{4}$ Hiroaki Masubuchi, ${ }^{5}$ \\ Shuichi Hagiwara, ${ }^{5}$ Kiyohiro Ohshima, ${ }^{5}$ Yuichi Iino, ${ }^{5}$ \\ Toru Takahashi ${ }^{6}$ and Izumi Takeyoshi ${ }^{6}$
}

\begin{abstract}
A 62-year-old man was transported to the emergency medical center because of blunt chest trauma combined with multiple trauma after a car accident. He complained of severe back pain. Contrastenhanced computed tomography revealed left hemopneumothorax with multiple left rib fractures, a scapular fracture, lung contusion, liver injury, right renal injury, and acute aortic dissection. Although open surgical repair and endovascular stent grafting were considered, primarily nonoperative management of the thoracic aortic injury under blood pressure control was selected because of the multiple trauma, type B aortic dissection, and oral administration of two antiplatelet drugs. The patient's hemodynamic and respiratory conditions remained stable in the intensive care unit, and the aortic dissection improved. We herein report a rare case of blunt thoracic aortic injury with oral administration of antiplatelet drugs resulting in multiple trauma and acute aortic dissection treated nonoperatively.

(Kitakanto Med J 2012; 62: 411 414)
\end{abstract}

Key words : traumatic aortic dissection, antiplatelet drug, multiple trauma

\section{Introduction}

Blunt traumatic injury and acute dissection of the thoracic aorta is increasing in incidence in patients with serious multitrauma and remains highly lethal. Early identification and treatment is key to a successful outcome. For treatment purposes, aortic dissections are usually classified as those involving the ascending aorta (type A dissections), which are usually managed surgically, and type B traumatic dissections, which are usually managed with endoluminal aortic stents. However, primarily nonoperative management of the thoracic aortic injury under blood pressure control should be selected in certain cases. We herein report a case of acute aortic dissection due to blunt thoracic aortic injury resulting in multiple trauma for which we selected primarily nonoperative management.

1 Department of Intensive Care, National Hospital Organization Takasaki General Medical Center, 36 Takamatsu-cho, Takasaki, Gunma 370-0829, Japan 2 Department of Surgery, Gunma University Graduate School of Medicine, 3-39-22 Showa-machi, Maebashi, Gunma 371-8511, Japan 3 Department of Cardiovascular Surgery, Gunma University Graduate School of Medicine, 3-39-22 Showa-machi, Maebashi, Gunma 371-8511, Japan 4 Department of Respiratory Surgery, Gunma University Graduate School of Medicine, 3-39-22 Showa-machi, Maebashi, Gunma 371-8511, Japan 5 Department of Emergency Medicine, Gunma University Graduate School of Medicine, 3-39-22 Showa-machi, Maebashi, Gunma 371-8511, Japan 6 Department of Thoracic and Visceral Organ Surgery, Gunma University Graduate School of Medicine, 3-39-22 Showa-machi, Maebashi, Gunma 371-8511, Japan

Received: July 19, 2012

Address: TAKASHI OGINO Department of Intensive Care, National Hospital Organization Takasaki General Medical Center, 36 Takamatsu-cho, Takasaki, Gunma 370-0829, Japan 


\section{Case report}

A 62-year-old man who did not routinely wear a seat belt in the rear seat was thrown out of the car when it crashed into the median strip. He was transported to our hospital within 60 minutes of the accident. On arrival in the emergency room, the patient had a blood pressure of $147 / 71 \mathrm{mmHg}$, a heart rate of 84 beats/minute, a respiratory rate of 16 breaths/ minute, and a Glasgow Coma Scale score of 15 (E4; V5; M6). He complained of severe back pain and had excoriations on the dorsal thorax and right forearm and shoulder. No findings suggesting visceral or appendicular ischemia were apparent.

His hemoglobin concentration was $15.4 \mathrm{~g} / \mathrm{dL}$, serum aspartate aminotransferase was $355 \mathrm{IU} / \mathrm{L}$, serum alanine aminotransferase was $298 \mathrm{IU} / \mathrm{L}$, serum lactate dehydrogenase was $860 \mathrm{IU} / \mathrm{L}$, serum creatine phosphokinase was $608 \mathrm{IU} / \mathrm{L}$, and serum creatinine was 1.28 $\mathrm{mg} / \mathrm{dL}$, all of which were increased from near their normal ranges. Contrast computed tomography (CT) revealed left hemopneumothorax with multiple left rib fractures, a scapular fracture, lung contusion (Fig. 1), liver injury, right renal injury (Fig. 2), and acute aortic dissection from the distal arch to the infrarenal abdominal aorta with no evidence of aortic rupture (Fig. 3). $\mathrm{He}$ had a history of angina and hypertension and was taking a hypertensive drug (amlodipine besylate, 10 $\mathrm{mg} /$ day) and two antiplatelet drugs (clopidogrel sulfate, $75 \mathrm{mg} /$ day and aspirin, $100 \mathrm{mg} /$ day).

The patient was admitted to the intensive care unit (ICU) and received mechanical ventilator support and nonoperative management by means of blood pressure control $(\leq 120 \mathrm{mmHg})$ using the antihypertensive drug nicardipine hydrochloride for 5 days. A 28-Fr thoracostomy tube was inserted into the right thoracic cavity, and extraction of the right thoracostomy tube was performed on the third day. The patient's hemodynamic and respiratory conditions remained stable in the ICU. On day 5 after admission, CT without contrast enhancement revealed no change in the size of the aortic dissection. Although slight renal

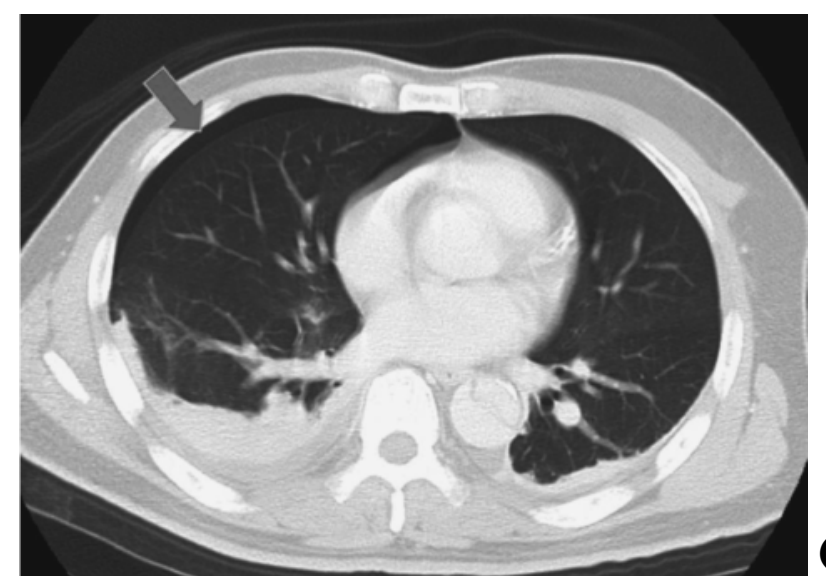

(a)

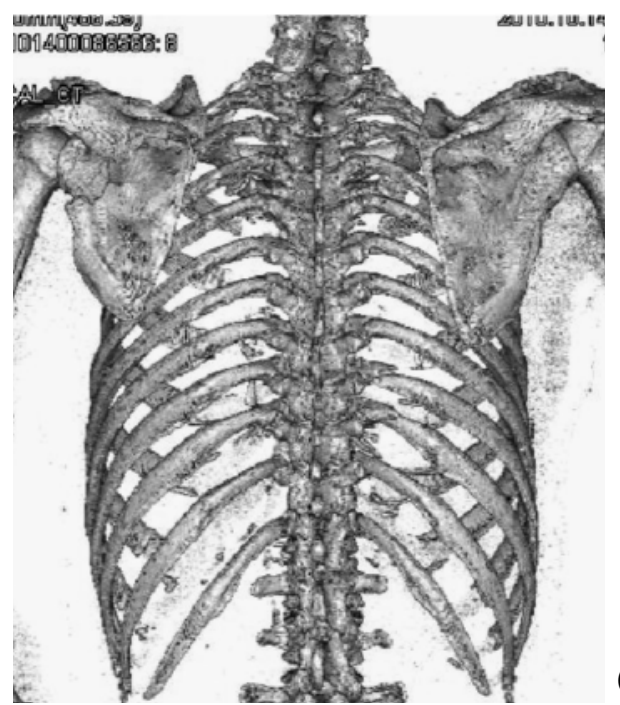

(b)

Fig. 1 Chest CT demonstrates left hemopneumothorax (a) with multiple left rib fractures, a scapular fracture, and lung contusion (b)

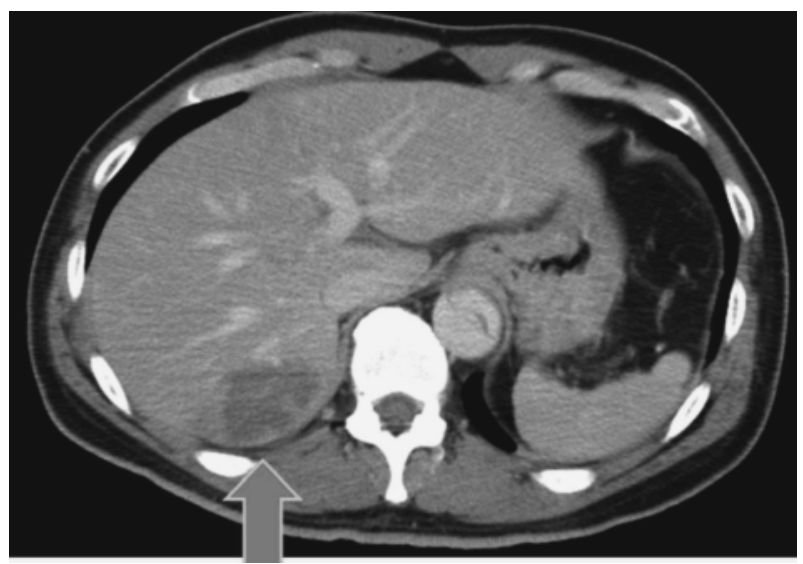

(a)

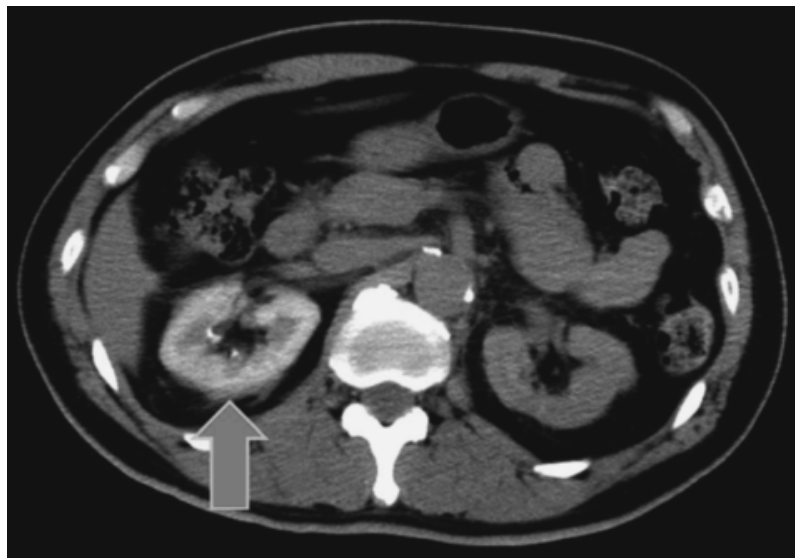

(b)

Fig. 2 Abdominal CT demonstrates (a) liver and (b) renal injuries 


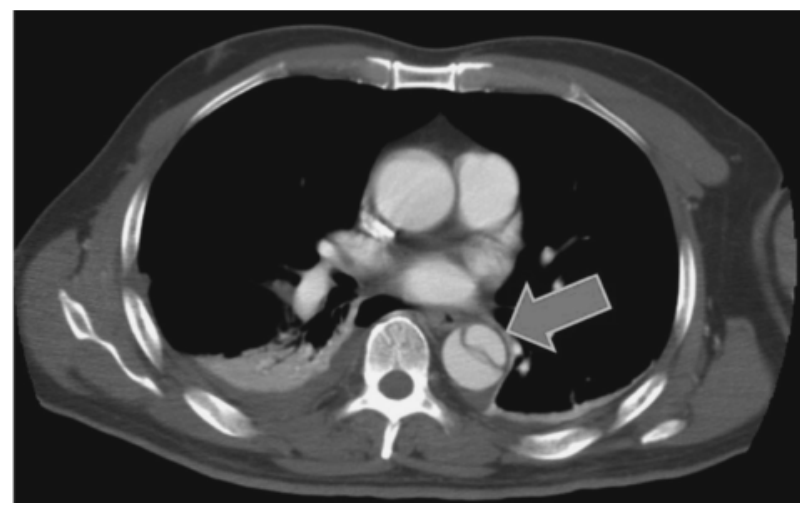

(a)

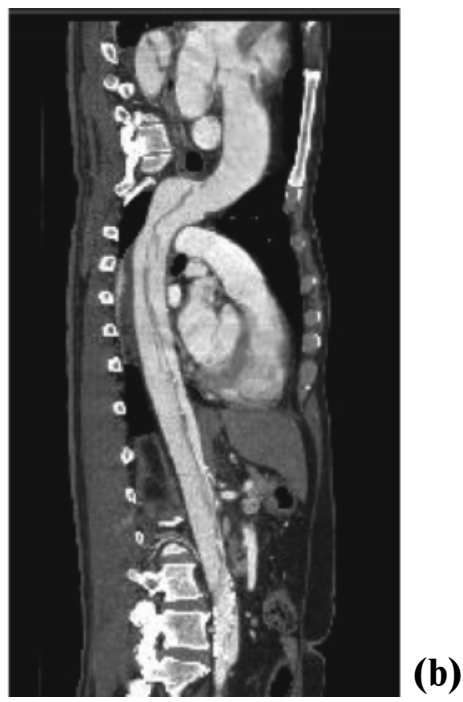

Fig. 3 Contrast-enhanced CT demonstrates acute aortic dissection from the distal arch to the infrarenal abdominal aorta (a), with no evidence of aortic rupture (arrows indicate the dissection and false lumen) (b)

disorder remained, the patient's clinical course was uneventful after discharge from the ICU on day 8 , and he was transferred to a different hospital for rehabilitation on day 42.

\section{Discussion}

Acute traumatic injury of the thoracic aorta is a relatively common injury in deceleration accidents, usually high-speed motor vehicle accidents, and is highly lethal. ${ }^{1,2}$ As a consequence of major car crashes, thoracic aortic ruptures occur in $10 \%$ to $30 \%$ of multiply injured patients. They represent the second most common cause of death after trauma, following only head injuries. Before hospital admission, the primary cause of death is hemorrhage, and only a small percentage of patients who actually receive medical attention survive. ${ }^{3,4}$ A few of these patients reach the hospital alive, and their management is further complicated by injuries of skeletal and visceral organs and a history of oral administration.

Clinical signs are not sensitive or specific, and chest X-rays may not identify the problem in a high number of multiply injured patients. The most important factor for successful hospital management of these patients is early diagnosis with multislice CT and appropriate urgent treatment, which may lead to a survival rate of $80 \% .^{5}$ The diagnosis and management of blunt thoracic aortic injuries have seen significant changes over the last decade. Although the outcome of aortic dissection due to blunt chest trauma is not always favorable, survival has improved. The most striking changes in the definitive management of thoracic aortic injuries are endovascular stent graft repair and nonoperative management by means of blood pressure control. ${ }^{6}$ For treatment purposes, aortic dissections are usually classified as those involving the ascending aorta (type A dissections), which are usually managed surgically, and type B traumatic dissections, which are usually managed with endoluminal aortic stents. ${ }^{7,8}$ Primarily nonoperative management of the thoracic aortic injury under blood pressure control should be selected in certain cases.

The concept of nonoperative management in selected high-risk, elderly patients with small aortic tears has shown encouraging results in early exploratory studies and has been suggested to be a reasonable alternative treatment in carefully selected patients. Acute aortic dissection is an uncommon presentation of blunt injuries of the thoracic aorta in the hospital, and the poor prognosis has not been adequately emphasized. When traumatic aortic dissection has been diagnosed with CT, it is important to bear in mind that it can become complicated by not only exsanguinating rupture, but also by splanchnic ischemia, as can nontraumatic dissection. We should select a nonoperative method of treatment for traumatic aortic dissection.

\section{Conclusion}

Early diagnosis and treatment of an aortic dissection in a multiply injured patient is often difficult, but is also particularly important for a favorable outcome. We should select open surgical repair, endovascular stent grafting, and nonoperative management for traumatic aortic dissection in patients with multiple trauma with consideration of the state of the patient. Early identification of all possible injuries and their thorough clinical and radiological investigation are of paramount importance for an effective treatment approach and a favorable clinical outcome.

\section{References}

1. Butterworth RJ, Thomas DJ, Wolfe JH, et al. Endovascular treatment of carotid dissecting aneurysms. Cerebrovascular Dis 1999; 9 : 241-247.

2. Liener UC, Sauerland S, Knoferl MW, et al. Emergency surgery for chest injuries in the multiply injured: A system- 
atic review. Unfallchirurg 2006; 109: 447-452.

3. Zoffoli G, Saccani S, Larini P, et al. Endovascular treatment of traumatic aortic dissection and innominate artery pseudoaneurysm. J Trauma 2006; 61: 447-450.

4. Hemmila MR, Hirschl RB, Teitelbaum DH, et al. Thracheobronchial avulsion and associated innominate artery injury in blunt trauma: case report and literature review. J Trauma 1999; $46: 505-512$.

5. Bingol H, Iyem H, Akay HT, et al. Endovascular repair in management of thoracic aortic aneurysms. Int $\mathrm{J}$ Cardiovasc Imaging 2006; 23 : 53-59.

6. Kushimoto S, Shiraishi S, Miyauchi M, et al. Visceral ischemia caused by acute aortic dissection following blunt aorticinjury: Report of a case. J Nippon Med Sch 2011 ; 78: 110-115.

7. Aziz F, Penupolu S, Alok A, et al. Peripartum acute aortic dissection: A case report \& review of literature. J Thorac 2011; 3: 65-67.

8. Demetriades D, Velmahos GC, Scalea TM, et al. Operative repair or endovascular stent graft in blunt traumatic thoracic aortic injuries: results of an American Association for the Surgery of Trauma Multicenter Study. J Trauma 2008 ; 64 : 561-571. 